Bull. Austral. Math. Soc.

Vol. 44 (1991) [375-380]

\title{
LOCAL INTEGRABILITY OF CR-HYPERSURFACES
}

\author{
Kil H. Kwon and Byeong C. Song
}

By characterising CR-hypersurfaces of dimension $m$ having $m$ strongly independent CR-functions, we find the necessary condition for a CR-hypersurface to be locally integrable and show that the same condition is also sufficient if the CR-hypersurface is strongly pseudoconvex and has $m$ strongly independent CRfunctions.

\section{Preliminaries}

Let $V$ be a CR-hypersurface of dimension $m(\geqslant 1)$ in an open set $\Omega$ in $\mathbf{R}^{2 m+1}$. That is, $V$ is a subbundle of $\mathbf{C} \otimes T \Omega$ of fibre dimension $m$ such that

$$
\begin{gathered}
{[V, V] \subset V} \\
V \cap \bar{V}=\{0\}(V \text { is "the complex conjugate" of } V) .
\end{gathered}
$$

We call $V$ locally integrable at $p$ in $\Omega$ if in some neighborhood $U$ of $p$ in $\Omega$ there are $m+1 C^{\infty}$-functions $\left\{u_{j}\right\}_{1}^{m+1}$ such that

(1.3) $u_{j}$ are CR-functions, that is, $L u_{j}=0$ for any smooth section $L$ of $V$ on $U$;

$$
d u_{1} \wedge \cdots \wedge d u_{m+1} \neq 0 \text {. }
$$

Let $V^{\prime}$ be the orthogonal of $V$ in $\mathbf{C} \otimes T^{*} \Omega$. Then the condition (1.2) is equivalent to $V^{\prime}+\bar{V}^{\prime}=\mathbf{C} \otimes T^{*} \Omega$. Hence, if $V$ is locally integrable, then there are $m$ CR-functions $\left\{u_{j}\right\}_{1}^{m}$ such that

$$
d u_{1} \wedge \cdots \wedge d u_{m} \wedge d \bar{u}_{1} \wedge \cdots \wedge d \bar{u}_{m} \neq 0
$$

We call $m$ such CR-functions to be strongly independent. Not every CR-hypersurface is locally integrable (see for example $[3,5,8,9]$ ) (even if it has $m$ strongly independent CR-functions). In [8], Nirenberg constructed a single $C^{\infty}$-vector field in $\mathbf{R}^{3}$

$$
L=\frac{\partial}{\partial \bar{z}}+i z \frac{\partial}{\partial t}+i z \phi(x, y, t) \frac{\partial}{\partial t}, \quad(\phi \text { is real-valued })
$$

Received 20 November 1990

Copyright Clearance Centre, Inc. Serial-fee code: 0004-9729/91 \$A2.00+0.00. 
satisfying for any $C^{\infty}$-function $\psi(x, y, t)$ with $L \psi=0, \partial \psi / \partial \bar{z}$ and $\partial \psi / \partial t$ vanish of infinite order at the origin. Thus the vector field $L$ spans a CR-hypersurface of dimension 1 having a strongly independent CR-function $z$, which is also strongly pseudoconvex (that is, $L, \bar{L}$, and $[L, \bar{L}]$ are linearly independent) but not locally integrable at the origin. Motivated by the above example, Hanges [2] found a necessary condition for a CR-hypersurface of dimension 1 to be locally integrable and showed that the same condition is also sufficient in some restricted cases. Here, we shall extend the results in [2] to the CR-hypersurfaces of any dimension $m \geqslant 1$.

Once we characterise the CR-hypersurfaces having $m$ strongly independent CRfunctions in terms of local coordinates (see for example, Proposition 2.1), the extension from $m=1$ to any $m \geqslant 1$ is rather straightforward.

\section{MaIN RESULTS}

From now on, $V$ always means a CR-hypersurface of dimension $m \geqslant 1$ in $\Omega$.

Proposition 2.1. Locally $V$ has $m$ strongly independent $C R$-functions if and only if $\Omega$ can be covered by local charts $(U ; x, y, t)$ in which $V$ is spanned by $m$ smooth vector fields

$$
L_{j}=\frac{\partial}{\partial \bar{z}_{j}}+a_{j}(x, y, t) \frac{\partial}{\partial t}, \quad z_{j}=x_{j}+i y_{j}, \quad j=1, \cdots, m .
$$

Proof: The sufficiency is clear since $z_{1}, \cdots, z_{m}$ are strongly independent CRfunctions of $V$. Conversely, let $u_{1}, \cdots, u_{m}$ be strongly independent CR-functions about a point, say 0 , in $\Omega$; then condition (1.5) is equivalent to

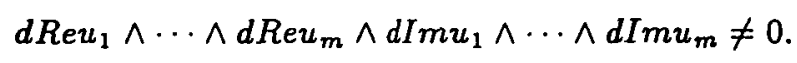

Hence we can take a local coordinate system $(x, y, t)$ about 0 where

$$
x_{1}=R e u_{1}, \cdots, x_{m}=R e u_{m}, y_{1}=I m u_{1}, \cdots, y_{m}=I m u_{m} .
$$

For any local basis $B=\left\{L_{j}\right\}_{1}^{m}$ of $V$ about 0 , we have

$$
L_{j}=\sum_{k=1}^{m} 2 L_{j}\left(R e u_{k}\right) \frac{\partial}{\partial \overline{z_{k}}}+L_{j}(t) \frac{\partial}{\partial t}, z_{j}=x_{j}+i y_{j}, j=1, \cdots, m .
$$

Note that the matrix $A=2\left[L_{j}\left(\operatorname{Re} u_{k}\right)\right]_{j, k}$ is nonsingular at 0 and so in some neighbourhood of 0 . Indeed, if otherwise, the vector fields $\left\{L_{j}-L_{j}(t) \partial / \partial t\right\}_{1}^{m}$ are linearly dependent at 0 and so $\partial / \partial t$ must be in $V \cap \bar{V}$ at 0 , which contradicts to (1.2). Then $A^{-1} B$ is another local basis of $V$ in a neighbourhood of 0 having the form (2.1). 
Note that when $V$ is spanned by $L_{j}$ in (2.1), condition (1.1) is equivalent to

$$
\left[L_{j}, L_{k}\right]=0 \text {, that is, } L_{j} a_{k}-L_{k} a_{j}=0, j, k=1, \cdots, m .
$$

Proposition 2.2. $V$ is locally integrable at $p$ in $\Omega$ if and only if there is a local chart $(U ; x, y, t)$ about $p$ in which $V$ is spanned by vector fields $L_{j}$ in (2.1) and a $C R$-function $u \in C^{\infty}(U)$ of the form

$$
u(x, y, t)=t+i \phi(x, y, t)
$$

where $\phi \in C^{\infty}(U)$ is real-valued and $\phi(p)=0$ and $d \phi(p)=0$.

Proof: The necessity is trivial since $\left\{z_{1}, \cdots, z_{m}, u\right\}$ are $m+1$ independent CRfunctions of $V$ on $U$. Conversely, by Proposition 2.1, locally, $V$ is spanned by $L_{j}$ in (2.1). Furthermore, we may assume $a_{j}(p)=0$ via suitable linear change of coordinates fixing $x$ and $y$. Then $\left\{z_{j}\right\}_{1}^{m}$ are $m$ independent CR-functions of $V$ on $U$. Since $V$ is locally integrable, there is a CR-function $u \in C^{\infty}(U)$ (possibly after shrinking $U$ ) such that

$$
d z_{1} \wedge \cdots \wedge d z_{m} \wedge d u \neq 0
$$

Then $(\partial u / \partial t)(p) \neq 0$, since otherwise $d u$ is a linear combination of $\left\{d z_{j}\right\}_{1}^{m}$ which contradicts (2.7). If we set $v=A u+\sum_{j=1}^{m} B_{j} z_{j}$ where $A=(\partial u / \partial t)(p)^{-1}$ and $B_{j}=$ $-\left(\partial u / \partial z_{j}\right)(p)\{(\partial u / \partial t)(p)\}^{-1}$, then $v$ is a CR-function satisfying $d v(p)=d t$. Hence $v(x, y, t)=t+W(x, y, t)$ with $d W(p)=0$ so that $\partial \operatorname{Rev} / \partial t(p) \neq 0$. Then in a new coordinate system $\left(x, y, t^{\prime}\right)$ with $t^{\prime}=t+\operatorname{Re} W$ about $p$, we have $v=t^{\prime}+i \phi\left(x, y, t^{\prime}\right)$ and $d \phi(p)=0$. We may assume $\phi(p)=0$.

For later use, we note that $a_{j}$ and $\phi$ are related by

$$
i \frac{\partial \phi}{\partial \bar{z}_{j}}+a_{j}\left(1+i \frac{\partial \phi}{\partial t}\right)=0, j=1, \cdots, m .
$$

We say that a smooth function $a(x, y, t)$ defined near a point $p$ in $\mathbf{R}^{2 m+1}$ is microanalytic at $(p,+1)$ (relative to $t$ ) (respectively $(p,-1)$ ) if there exist a neighbourhood $\mathcal{O}$ of $(p, 0)$ in $\mathbf{R}^{2 m+2}$ and $A(x, y, t, s) \in C^{\infty}(\mathcal{O})$ such that

$$
\begin{gathered}
\frac{\partial A}{\partial \bar{w}}=0 \text { in } \mathcal{O}^{+}\left(\text {respectively } \mathcal{O}^{-}\right) \\
A(x, y, t, 0)=a(x, y ; t) \text { in } \mathcal{O}^{0}=\left\{(x, y, t) \in \mathbf{R}^{2 m+1}:(x, y, t, 0) \in \mathcal{O}\right\}
\end{gathered}
$$

where $w=t+i s$ and $\mathcal{O}^{+}\left(\mathcal{O}^{-}\right)=\{(x, y, t, s) \in \mathcal{O}: s>0(s<0)\}$. 
TheOREM 2.1. (Necessity) If $V$ is locally integrable in $\Omega$, then for any $p$ in $\Omega$ there is a local chart $(U ; x, y, t)$ about $p$ in which $V$ is spanned by the vector fields $L_{j}$ in $(2.1)$, where all $a_{j}$ are microanalytic at $(p,+1)$.

We shall omit the proof since it is essentially the same as that of Theorem 1 in [2].

For a local basis $\left\{L_{j}\right\}$ of $V$ and any real vector field $L_{0} \notin V+\bar{V}$ about a point $p$ in $\Omega$, define complex numbers $C_{j, k}(p)$ by

$$
-\frac{i}{2}\left[L_{j}, \bar{L}_{k}\right](p)-C_{j, k}(p) L_{0}(p) \in V_{p}+\bar{V}_{p}
$$

We call the $m \times m$ matrix $L(p)=\left[C_{j, k}(p)\right]_{j, k}$ the Levi matrix of $V$ at $p$. When it is nonsingular, the absolute value of its signature is an invariant of $V$. We call $V$ strongly pseudoconvex at $p$ if $L(p)$ is positive or negative definite.

Theorem 2.2. (Sufficiency) Let $V$ be a CR-hypersurface of dimension $m$ having $m$ strongly independent $C R$-functions so that $V$ has a local basis $\left\{L_{j}\right\}_{1}^{m}$ in (2.1). Then $V$ is locally integrable at $p$ in $\Omega$ if the Levi matrix $L(p)$ of the system $\partial / \partial t, L_{1}, \cdots, L_{m}$ is positive (respectively negative) definite at $p$ and the coefficients $a_{j}$ of $L_{j}$ are microanalytic at $(p,+1)$ (respectively $(p,-1)$ ). In this case, there is a CR-function $u$ of the form (2.6) which is also microanalytic at $(p,+1)$ (respectively $(p,-1))$.

We need the following lemma which can be easily proved by Proposition 2.2 (see for example, [2]).

Lemma . Let $V$ be the same as in Theorem 2.2. Then $V$ is locally integrable at $p$ in $\Omega$ if and only if there exist an open neighbourhood $U$ of $p$ and $v \in C^{\infty}(U)$ satisfying

$$
L_{j} v=-\frac{\partial a_{j}}{\partial t}, \quad j=1, \cdots, m .
$$

Proof of Theorem 2.2: We always assume $p=0$. Since $a_{j}$ and so $f_{j}:=$ $-\partial a_{j} / \partial t$ are microanalytic at $(0,+1)$, we have an open neighbourhood $\mathcal{O}$ of 0 in $\mathbf{R}^{2 m+2}$ and $A_{j}, F_{j} \in C^{\infty}(\mathcal{O})$ such that

$$
\begin{gathered}
\frac{\partial A_{j}}{\partial \bar{w}}=\frac{\partial F_{j}}{\partial \bar{w}}=0 \quad \text { in } \mathcal{O}^{+}, \\
A_{j}(x, y, t, 0)=a_{j}(x, y, t), F_{j}(x, y, t, 0)=f_{j}(x, y, t) \text { in } \mathcal{O}^{0} .
\end{gathered}
$$

On $\mathcal{O}^{+}$, define $L_{0}^{+}=\partial / \partial \bar{w}$ and $L_{j}^{+}=\partial / \partial \bar{z}_{j}+A_{j} \partial / \partial w, j=1, \cdots, m$. Then they are linearly independent and commute with each other. The latter comes directly from (2.5), (2.13) and Schwarz's reflection principle. Hence $\left\{L_{j}^{+}\right\}_{0}^{m}$ define a (almost) complex structure on $\mathrm{O}^{+}$by Newlander-Nirenberg theorem [7]. 
Now in order to solve the system (2.12), it suffices to solve

$$
L_{0}^{+} u=0, L_{j}^{+} u=F_{j}, j=1, \cdots, m
$$

smoothly up to $\{s=0\}$.

Let $\alpha_{0}, \cdots, \alpha_{m}$ be 1 -forms which are dual to $L_{0}^{+}, \cdots, L_{m}^{+}$(for example, $\alpha_{0}=$ $d \bar{w}-\sum_{1}^{m} \bar{A}_{j} d z_{j}$ and $\left.\alpha_{j}=d \bar{z}_{j}, j=1, \cdots, m\right)$. Then (2.15) becomes

$$
\bar{\partial} u=\sum_{j=1}^{m} F_{j} \alpha_{j}
$$

where the operator $\bar{\partial}$ is defined on $v \in C^{\infty}\left(\mathcal{O}^{+}\right)$by $\bar{\partial} v=\sum_{j=0}^{m}\left(L_{j}^{+} v\right) \alpha_{j}$.

Using (2.3), (2.13) and Schwarz's reflection principle, we can see that the 1 -form $\sum_{j=1}^{m} F_{j} \alpha_{j}$ is $\bar{\partial}$-closed, that is, $\bar{\partial}\left(\sum_{j=1}^{m} F_{j} \alpha_{j}\right)=0$.

As in [2], we can find a strongly pseudoconvex domain in $\mathcal{O}^{+}$whose boundary intersects with $\{s=0\}$ near 0 . Hence, by the well known fact about the $\bar{\partial}$-problem (see for example, $[1]$ ), we can solve the equation (2.16) (or equivalently (2.15)) smoothly up to $\{s=0\}$.

REMARK. In fact, if $m \geqslant 4$, then Theorem 2.2 gives only a simple proof of a special case of Kuranishi's theorem [6] saying that any strongly pseudoconvex CR-hypersurface of dimension $\geqslant 4$ is locally integrable. However, for $m<4$ (at least for $m=1$ by Nirenberg's example in [8]) not every strongly pseudoconvex CR-hypersurface of dimension $m$ is locally integrable.

\section{ReFERENCES}

[1] G.B. Folland and J.J. Kohn, The Neumann problem for the Cauchy-Riemann complex (Princeton University Press, Princeton, N.J., 1972).

[2] N. Hanges, 'The missing first integral', J. Differential Equations 72 (1988), 178-188.

[3] H. Jacobowitz, 'Simple examples of non-realizable CR hypersurfaces', Proc. Amer. Math. Soc. 98 (1986), 467-468.

[4] H. Jacobowitz, 'The canonical bundle and realizable CR hypersurfaces', Pacific J. Math. 127 (1987), 91-101.

[5] H. Jacobowitz and F. Treves, 'Non-realizable CR structures', Invent. Math. 66 (1982), 231-249.

[6] M. Kuranishi, 'Strongly pseudoconvex CR structures over small balls', Ann. of Math. Part I 115 (1982), 451-500. Part II 116 (1982), 1-64. Part III 116 (1982), 249-330. 
[7] A. Newlander and L. Nirenberg, 'Complex analytic coordinates in almost complex manifolds', Ann. of Math. 65 (1957), 391-404.

[8] L. Nirenberg, Lectures on linear partial differential equations: Regional Conference Series in Mathematics (Amer. Math. Soc., Providence, R.I., 1973).

[9] L. Nirenberg, 'On a question of Hans Lewy', Russian Math. Surveys 29 (1974), 251-262.

Department of Mathematics

KAIST

P.O. Box 150

Cheongryang, Seoul

130-650 Korea 